\title{
二茂钛杂环化合物的合成及应用研究进展
}

\author{
王萍 章彦婧 王伟 郑卫新* \\ (杭州师范大学材料与化学化工学院 杭州 310036)
}

\begin{abstract}
摘要 钛杂环化合物分子内含有两个碳金属键，是一类重要的有机合成中间体. 综述了近年来二茂钛杂三元、四元、 五元、六元环化合物的结构特征、合成以及其在有机合成中的应用研究进展，就钛杂环中 $\mathrm{C}-\mathrm{Ti}$ 键切断的选择性及反 应活性开展了讨论与展望.
\end{abstract}

关键词 钛杂三元环; 钛杂四元环; 钛杂五元环; 钛杂六元环; 合成; 选择性反应

\section{Progresses on Synthesis and Applications of Bis(cyclopentadieny)titanacycles}

\author{
Wang, Ping Zhang, Yanjing Wang, Wei Zheng, Weixin* \\ (College of Material, Chemistry \& Chemical Engineering, Hangzhou Normal University, Hangzhou 310036)
}

\begin{abstract}
Titanacycles, containing two carbon metal bonds in the molecule, have emerged as a series of significant intermediates in organic synthesis. The structural characteristics, preparation and synthetic applications of bis(cyclopentadienyl)titanathree, four, five, or six-membered rings have been summarized in the present review, in which the selective cleavage and reactivity of $\mathrm{C}-\mathrm{Ti}$ bond in the titanacyle have been discussed.

Keywords titana-three-menbered ring; titana-four-menbered ring; titana-five-menbered ring; titana-six-menbered ring; preparation; selective reaction
\end{abstract}

金属杂环化合物是一类重要的有机金属化合物，广 泛应用于合成化学的多个领域, 其合成及应用是传统合 成方法学的一个重大突破. 该类分子中包含的多个活性 碳一金属键及其特有的环状结构可进一步转化, 为化学 键选择性断裂提供机遇, 具有高效、高选择性及多样化 的反应特性 ${ }^{[1]}$. 过渡金属催化或促进的不饱和化合物的 还原偶联反应是合成金属杂环化合物的典型反应之 - ${ }^{[2]}$.

钛是 IVB 族元素, 其核外电子层结构为 $[\mathrm{Ar}] 3 \mathrm{~d}^{2} 4 \mathrm{~s}^{2}$. 由于原子外层有空的 $\mathrm{d}$ 轨道, 钛试剂与电子供体底物配 位可活化对应的共价键, 使底物作为一类具有选择性的 碳负离子等当体, 在碳-碳键、碳一杂原子键形成中实现 高选择性反应而受到人们关注 ${ }^{[3]}$.

化学家对钛杂环化合物的研究源自 19 世纪 50 年 代 ${ }^{[4]}$, 无配体钛杂化合物 $\mathbf{1}^{[5]}$ “ $\mathrm{Cp} 2 \mathrm{Ti}(\mathrm{II}) ”\left(\mathrm{Cp}=\eta^{5}-\mathrm{C}_{5} \mathrm{H}_{5}\right)$
是具有 $\mathrm{d}^{2}$ 结构的 14 电子体系 ${ }^{[6]}$ ，尽管目前未被分离得 到, 但它作为一种具有缺电子中心的高活性物种, 在有 机反应中具有较高的应用价值. 例如, 能与一系列不饱 和化合物发生还原偶联反应形成二茂钛杂环化合物，其 结构中的碳-钛键可进一步发生多种转化, 是有机金属 杂环化学研究的重要组成部分 ${ }^{[7]}$.

Willkinson 等 ${ }^{[8]}$ 通过 $\mathrm{TiCl}_{2}$ 与 2 equiv. 的环戊二烯基 钠 $(\mathrm{CpNa})$ 反应合成化合物 $\mathbf{1}$ (Path a $)^{[9]}$. 此外, 在氢气、加 热或光照的条件下, $\left(\eta^{5}-\mathrm{C}_{5} \mathrm{H}_{5}\right)_{2} \mathrm{TiR}_{2}(\mathrm{R}=\mathrm{Cl}$ 或 $\mathrm{Me})$ 在活性 金属(䒬钠或金属钠)促进下也能生成化合物 1 (Path b) ${ }^{[10]}$ (Scheme 1).

1970 年 Watt 等 ${ }^{[11]}$ 用 X 射线衍射法确证了化合物 1 的结构, 2002 年 Gordon 和 Freitag 用现代计算法(HartreeFock 理论、从头算、DFT 理论)计算出化合物 $\mathbf{1}$ 的基态 结构 ${ }^{[12]}$.

* E-mail: wxzheng@hznu.edu.cn

Received October 10, 2013; revised December 30, 2013; published online January 10, 2014.

Project supported by the National Natural Science Foundation of China (No. 20972037), the Special Funds for Key Innovation Team of Zhejiang Province (No. 2010R50017), the Excellent Young Teacher Support Program of Hangzhou Normal University (No. 2011-01-013) and the Program for Changjiang Scholars and Innovative Research Team in Chinese University (No. IRT 1231).

国家自然科学基金(No. 20972037)、浙江省重点科技创新团队建设项目(No. 2010R50017)、杭州师范大学优秀中青年教师支持计划(No. HNUEYT 2011-01-013)、教育部创新团队 “长江学者和创新团队发展计划资助(No. IRT 1231)资助项目. 


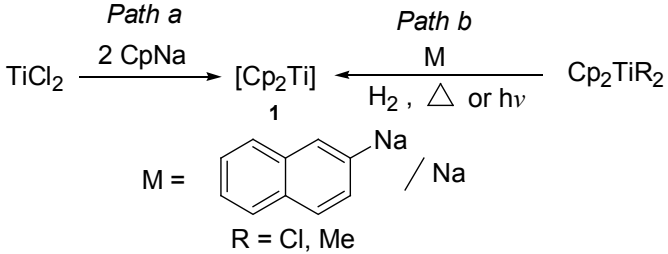

Scheme 1

自 20 世纪 50 年代 Ziegler-Natta 催化剂问世以来, 有机钛化合物得到了迅猛发展. 人们对钛化合物的反应 活性及其在有机合成中的应用开展研究, 发现有机钛作 为一类金属亲核试剂, 具有独特的化学选择性和立体选 择性 ${ }^{[13]}$. 其中常用的合成前体二氯二茂钛 $\left(\mathrm{Cp}_{2} \mathrm{TiCl}_{2}\right)$ 具 有制备方便、成本低廉、性质稳定、低毒高效等优点，应 用于多种合成反应中 ${ }^{[14]}$. 在不同的还原体系下, 以 $\mathrm{Cp}_{2} \mathrm{TiCl}_{2}$ 为底物可合成一系列钛杂环化合物 ${ }^{[15]}$.

根据环尺寸大小, 钛杂环可分为三元、四元、五

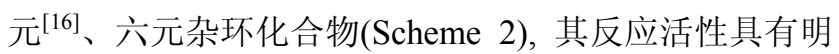
显的差异. 本文将介绍近年来几种常见的钛杂环化合物 的结构特征、合成及其在有机合成中的应用.
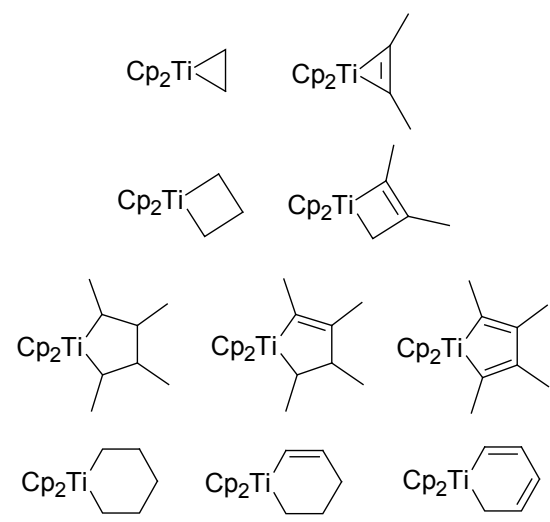

Scheme 2

\section{1 三元钛杂环}

三元钛杂环化合物主要有钛杂环丙烷和钛杂环丙 烯两类 ${ }^{[17]} . \mathrm{Cp}_{2} \mathrm{TiCl}_{2}$ 与 2 equiv. 金属还原剂(常用 $\mathrm{EtMgBr}^{[18]}$ 或 $\left.n-\mathrm{BuLi}^{[19]}\right)$ 反应生成的二烃基二茂铁 $\left(\mathrm{Cp}_{2} \mathrm{TiR}_{2}\right)$ 可通过 $\beta$-氢消除生成钛-烯烃 $\pi$-络合物 $\mathbf{2 , 2}$ 经 氧化加成形成 $\sigma$-络合物 3 即铁杂环丙烷 ${ }^{[20]}$ (Scheme 3). 其中 2 与 3 在反应体系中达到动态平衡, 该体系是 14 电子 “ $\mathrm{Cp}_{2} \mathrm{Ti}$ ” 活性物种的等当体, 是构建其他钛杂环的 反应前体及其他低价钛物种的重要来源.

Scheme 3 中 “ $\mathrm{Cp}_{2} \mathrm{Ti}$ ” 活性物种的等当体 2 与炔烃 发生配体交换即形成二茂钛与炔烃的 $\pi$-络合物结构 $\mathbf{4 , 4}$ 与炔烃经氧化加成生成钛杂环丙烯 $\mathbf{5}^{[21]}$ (Scheme 4). Nugent 等 ${ }^{[22]}$ 发现钛杂环丙烯具有两种结构, 一种是乙 炔的 $\pi$-络合物结构 4 (+2 价的氧化态), 另一种是 $\sigma$-络

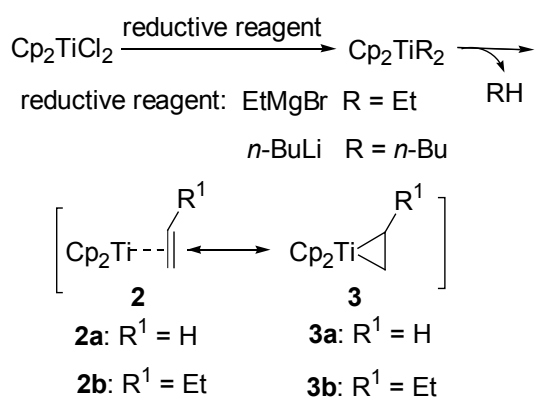

Scheme 3

合物结构 5 ( +4 价的氧化态). 该物种可与其他 $\pi$ 体系(如 炔烃、芳烃、烯烃等)发生插入反应生成五元钛杂环 ${ }^{[23]}$.

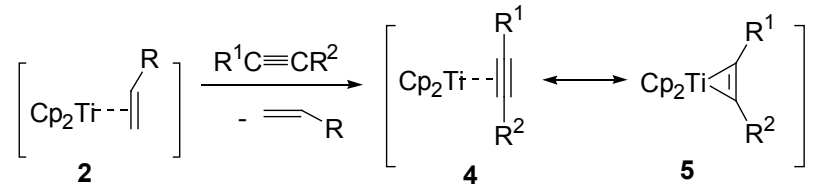

Scheme 4

在钛杂环丙烯类化合物中，2,3-二(三甲基硅基)-二 茂钛杂环丙烯化合物 6 是 “自由”二茂钛物种 ( “ $\mathrm{Cp}_{2} \mathrm{Ti}$ ”) 的一种稳定来源, 并能进行进一步的化学反应 ${ }^{[24]}$.

化合物 6 的合成主要有以下两种方法: (a)在 THF 中 以镁为还原剂, $\mathrm{Cp}_{2} \mathrm{TiCl}_{2}$ 与二 (三甲基硅基)乙炔 $\left(\mathrm{Me}_{3} \mathrm{SiC} \equiv \mathrm{CSiMe}_{3}\right)$ 的反应 ${ }^{[25]}$; (b) $\mathrm{Cp}_{2} \mathrm{TiMe}_{2}$ 与（二甲硅基 乙炔基)三甲基硅烷 $\left(\mathrm{Me}_{3} \mathrm{SiC}_{2} \mathrm{SiMe}_{2} \mathrm{H}\right)$ 在正己烷中的反 应 ${ }^{[26]}$ (Scheme 5).

(a) $\mathrm{Cp}_{2} \mathrm{TiCl}_{2}+\mathrm{Me}_{3} \mathrm{SiC} \equiv \mathrm{CSiMe}_{3}$

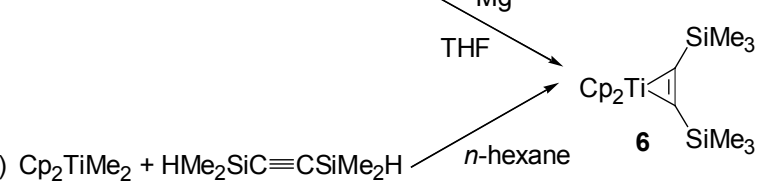

\section{Scheme 5}

化合物 6 中的硅具有空的 $\mathrm{d}$ 轨道, 可稳定负碳中心, 降低整个配合物的能量; 而硅作为供电子基团，可推动 钛环中的配体部分二-(三甲基硅基) 乙炔 $\left(\mathrm{Me}_{3} \mathrm{SiC} \equiv\right.$ $\mathrm{CSiMe}_{3}$ )离去产生活性钛物种 “ $\mathrm{Cp}_{2} \mathrm{Ti}$ ” , 在一定程度上 超越了炔烃的偶联反应。该化合物在有机合成中的应用 可能存在两种途径: (a) 通过配体解离-配位机理生成新 的金属物种(Path a); (b)通过配位-插入机理进行的炔烃 与底物协同反应(Path b $)^{[27]}$ (Scheme 6).

化合物 6 与炔烃反应因底物炔烃结构差异而不同. 当底物为对称内炔时，以二苯基乙炔 $(\mathrm{PhC} \equiv \mathrm{CPh})$ 为例， 化合物 6 中的二-(三甲基硅基)乙炔基可与二苯基乙炔 发生配体交换, 形成 2,3-二苯基钛杂环丙烯，当加入过 


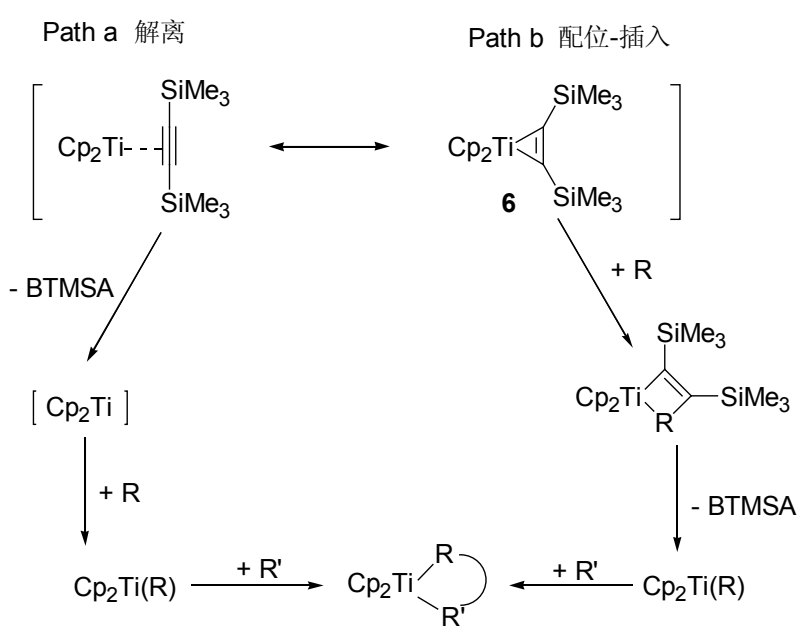

Scheme 6

量的二苯基乙炔时, 能得到对称的 2,3,4,5-四苯基-二茂 钛杂环成二烯(Scheme 7) ${ }^{[25 b]}$.

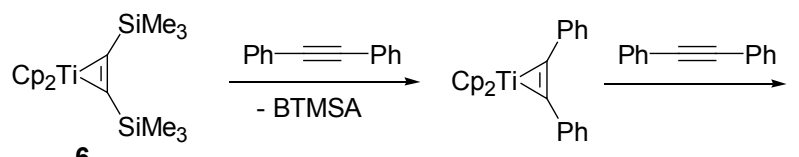

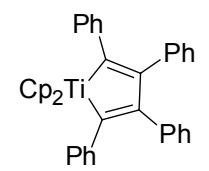

Scheme 7

6 与非对称炔基硅烷反应可通过配体交换-还原偶 联首先生成动力学产物 7 , 由于硅基对碳负离子的稳定 化作用使其进一步转化为热力学稳定的对称化合物 8 . 由于空间位阻因素, 无法获得另一个硅基处于 $\beta, \beta^{\prime}$-位的 对称结构化合物 ${ }^{[25 b, 28]}$, 从而实现高区域和立体选择性 控制(Scheme 8).

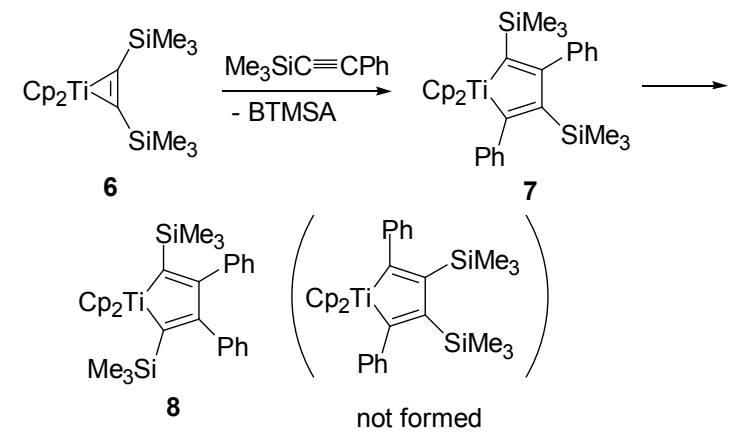

Scheme 8

端炔 $(\mathrm{RC} \equiv \mathrm{CH})$ 与 6 反应时, 6 中的二-(三甲基硅基) 乙炔基经配体交换形成端炔与钛的 $\pi$-配合物, 因炔碳上 质子具有酸性, 钛发生氧化加成反应生成炔基钛氢试 剂，与另一分子端炔发生钛氢化反应形成炔基烯基钛 9. 当上述步骤重复发生后可获得反式烯烃的聚合物 ${ }^{[27]}$.
当乙炔作为底物、反应温度控制在 $-20{ }^{\circ} \mathrm{C}$ 时，除了聚合 物外, 还有部分钛杂环戊二烯 10 生成(Scheme 9) ${ }^{[21,29]}$.

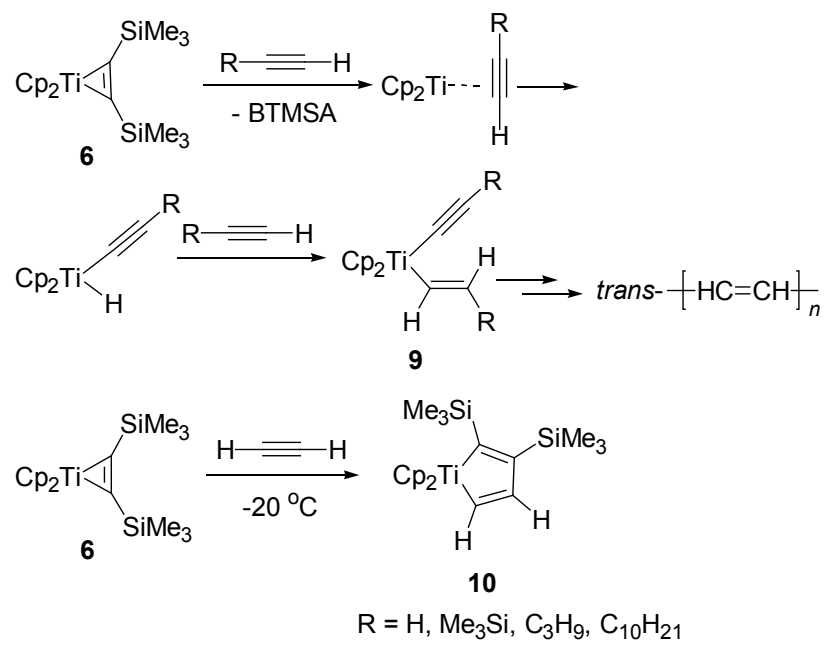

\section{Scheme 9}

从该部分反应中我们可以看出, 钛杂环丙烷与钛杂 环丙烯不仅是 14 电子 Ti(II)物种的来源，也是合成五元 钛杂环的重要前体. 在发生反应过程中底物烯或炔上取 代基对反应历程产生影响, 而温度也是重要的决定因素 之一.

\section{2 四元钛杂环}

近年来，IVB 族金属杂小环化合物因其反应活性及 稳定性而倍受关注. 四元钛杂碳环化从共价键类型上可 分为钛杂环丁烷 11、钛环丁烯 $12^{[5]}$ 及钛杂环丁二烯 13 (Scheme 10). 其中化合物 13 尽管至今尚未成功合成, 但是理论计算的研究结果已预测其存在的可能 ${ }^{[30]}$. 将 13 中构环碳原子替换成杂原子(如 $N, S$ 等)是稳定该类 化合物的重要策略 ${ }^{[31]}$.

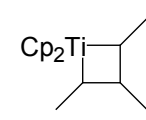

11

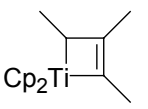

12<smiles></smiles>

13
Scheme 10

钛卡宾试剂与 $\pi$ 体系的加成及复分解反应是合成四 元钛杂环化合物的有效方法. 钛卡宾 14 可通过以下几 个底物的 $\beta$-消除反应来获得 ${ }^{[32]}$ : 1) Tebbe 试剂 ${ }^{[33]}$ 在 Lewis 碱作用下消除二甲基氯化铝; 2)钛杂环丁烷 15 热 分解；3) Petasis 试剂(二甲基二茂钛)中一个甲基的 $\alpha$-消 除 ${ }^{[34]}$ (Scheme 11). 作为前过渡金属卡宾 ${ }^{[35]}$, 钛卡宾中 $\alpha$-碳原子具有较强的亲核性, 可与亲电试剂反应 ${ }^{[36]}$, 在 一些化学计量反应以及催化反应(如羰基的烯化反

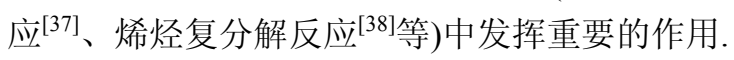




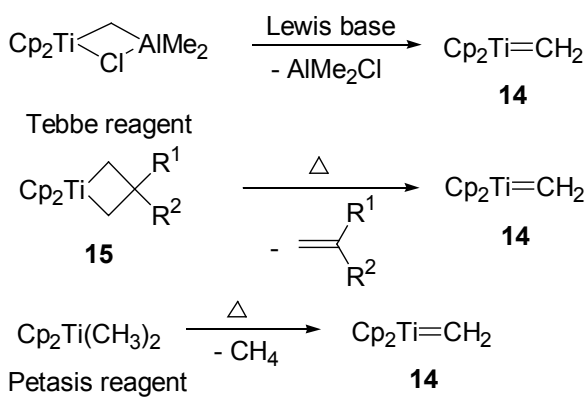

Scheme 11

\section{1 钛杂环丁烷}

钛卡宾与多种烯烃反应可构建性质各异的钛杂环 丁烷.

1)烃基取代的钛杂环丁烷：钛卡宾与 1,2-二取代烯 烃的双键发生插入反应形成钛杂环丁烷 11 (Eq. 1).

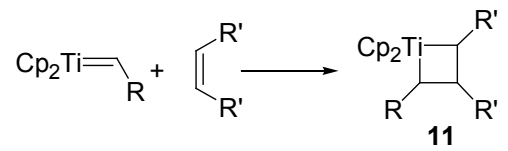

在上述反应中, 烯烃若是线性的, 产生的钛杂环丁 烷化合物具有较好的热力学稳定性; 而若烯烃是非线性 的, 反应得到的钛杂环丁烷化合物通常只在 $0{ }^{\circ} \mathrm{C}$ 以下 才能稳定存在 ${ }^{[39]}$.

钛卡宾亦可与环烯中的双键进行 [2+2]加成形成骈 环化合物 $16 、 17^{[40]}$, 经开环重排形成新的钛卡宾 (Scheme 12), 可再一次与新的不饱和体系发生复分解 反应 ${ }^{[41]}$. 由于环骨架的存在, 形成新卡宾结构的同时在 分子内产生一个新的碳碳双键.

2)亚甲基钛杂环丁烷：该类化合物是由钛卡宾与联 烯的插入反应制得的 ${ }^{[42]}$. 联烯中 $\mathrm{sp}$ 杂化的中心碳原子 与缺电子的钛相连，在取代基少的一端形成 $\alpha$-亚甲基钛

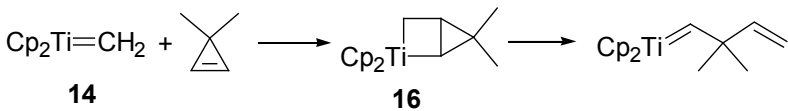

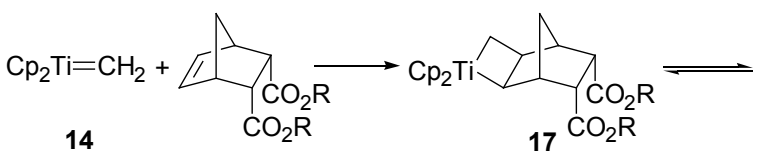

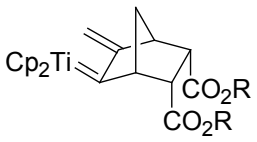

Scheme 12

杂环丁烷 18. 形成 18 的立体化学受温度影响较为明显, 当 $\mathrm{R}^{1}=\mathrm{Me}, \mathrm{R}^{2}=i$ - $\mathrm{Pr}$ 时, 在室温下产物以 $18 \mathrm{a}$ 为主，而 当温度升高至 $76{ }^{\circ} \mathrm{C}$ 产物则以 $\mathbf{1 8 b}$ 为主 ${ }^{[42 \mathrm{a}]}$. 该物种可消 除一分子乙烯生成新的烯钛卡宾 19 , 与炔烃通过插入 反应生成 $\alpha$-亚甲基钛杂环丁烯 20 , 与醛、酮经复分解反 应分别形成三取代、四取代联烯 ${ }^{[42 c]}$ (Scheme 13).

\section{2 钛杂环丁烯}

钛卡宾与炔烃反应可高区域选择性生成相应的钛 杂环丁烯 $12^{[5,23,43]}$ (Eq. 2).

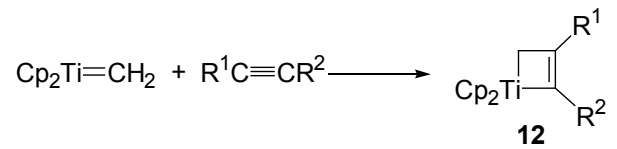

化合物 12 中的钛中心分别与 $\mathrm{C}_{\mathrm{sp}^{3}}$ 和 $\mathrm{C}_{\mathrm{sp}^{2}}$ 相连，所形 成的碳钛键可发生选择性切断. 例如，与羰基化合物发 生插入反应的区域选择性受到取代基效应的影响. 如图 16 所示, 当 12 中 $\mathrm{R}^{1}$ 为 $\mathrm{Ph}$ 时, 羰基插入 $\mathrm{C}_{\mathrm{sp}^{3}}$ 一钛键中形 成钛杂氧杂六元环 $21^{[44]}$; 若 $\mathrm{R}^{1}$ 为烷基时, 羰基则插入 到 $\mathrm{C}_{\mathrm{sp}^{2}}$ 一钛键中生成六元杂环 $22^{[45]}$. 产物中双键的构型 受制于反应中形成的环骨架而得以控制，可用于多取代 烯丙醇或高烯丙醇的选择性合成(Scheme 14).
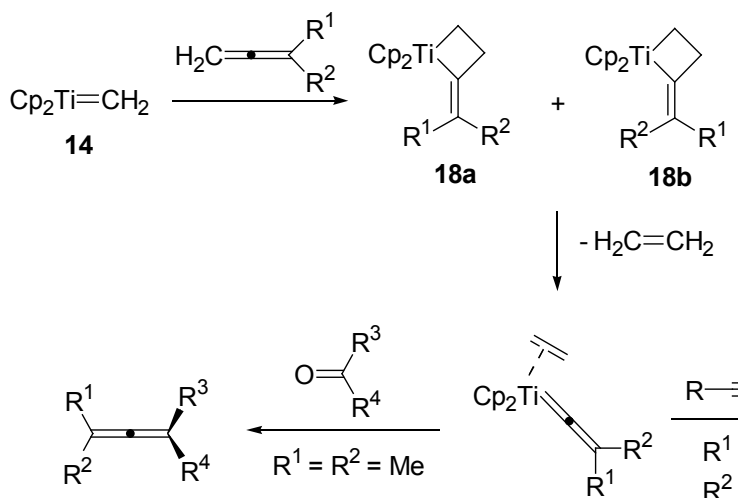

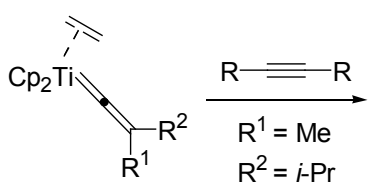

19

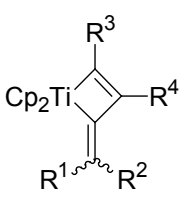

20

Scheme 13 


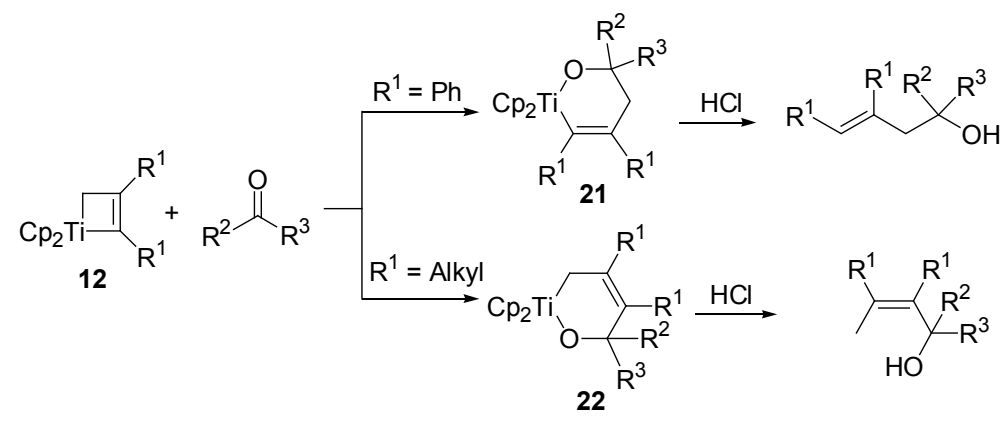

Scheme 14

Takeda 等 ${ }^{[46]}$ 通过二价钛物种 $\mathrm{Cp}_{2} \mathrm{Ti}\left[\mathrm{P}(\mathrm{OEt})_{3}\right]_{2}$ 与偕 二氯环丙烷的反应合成环丙烷钛卡宾 23,23 与内炔作用 形成螺环状钛杂环丁烯 24, 可用于立体选择合成系列环 丙基烯烃. 当结构中含二环 [4.1.0]庚烷骨架时钛杂环丁 烯 24 具有令人意外的稳定性, 无需无水无氧条件即可 分离获得, 其结构经单晶衍射确认 ${ }^{[47]}$ (Scheme 15).
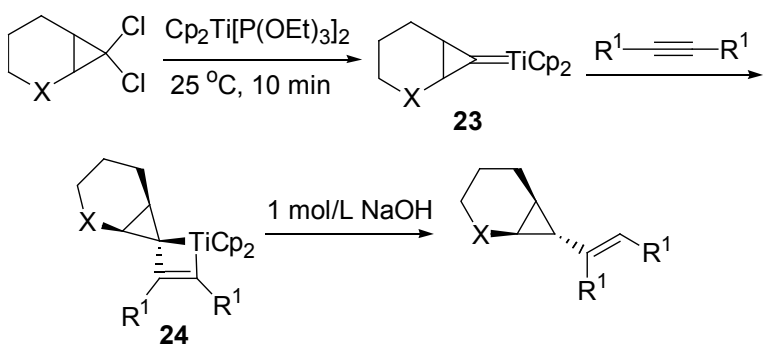

\section{Scheme 15}

在上述反应中, $\mathrm{Cp}_{2} \mathrm{Ti}\left[\mathrm{P}(\mathrm{OEt})_{3}\right]_{2}$ 可在室温下即可反 应, 与烯烃配合物 2 相比, 反应条件更温和. $\mathrm{Cp}_{2} \mathrm{Ti}\left[\mathrm{P}(\mathrm{OEt})_{3}\right]_{2}$ 在钛杂环丁烯的合成中发挥重要的作用, 在 Scheme 16 的反应中, 其与碳酸炔丙酯经氧化加成生 成联烯基钛(IV)物种 25,25 中与钛相连的双键与第二分 子 $\mathrm{Cp}_{2} \mathrm{Ti}\left[\mathrm{P}(\mathrm{OEt})_{3}\right]_{2}$ 形成亚甲基钛杂环丙烷 26, 26 在氯代 烃作用下经开环、还原消除形成钛卡宾 26 . 因 26 与经

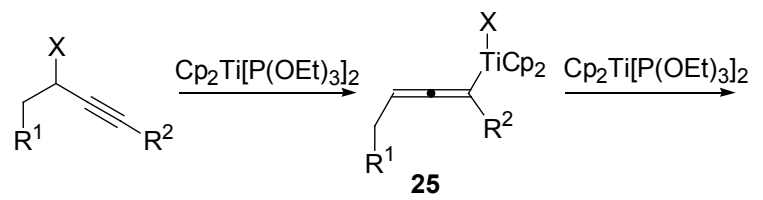

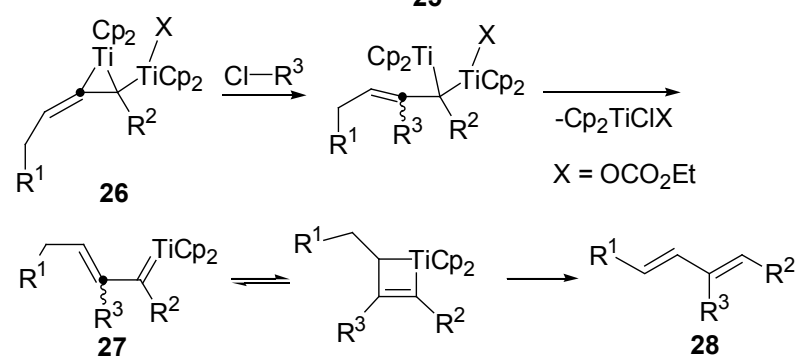

Scheme 16
分子内钛卡宾与双键的插入而生成的钛杂环丁烯之间 存在着动态平衡 ${ }^{[48]}$, 产物共轭二烯 28 中 1-位双键的构 型得以控制 ${ }^{[49]}$.

钛卡宾与 $\pi$ 体系的插入反应是合成钛杂四元环的重 要途径. 其中钛卡宾与联烯的反应可形成同时含有 $\mathrm{C}_{\mathrm{sp}^{3}}$ 一钛和 $\mathrm{C}_{\mathrm{sp}^{2}}$ 一钛键的 $\alpha$-亚甲基钛杂环丙烷，该物种脱 去一分子乙烯产生联烯钛卡宾. 19 与炔烃反应形成的 $\alpha-$ 亚甲基钛杂环丙烯 20 骨架中包含两个 $\mathrm{C}_{\mathrm{sp}^{2}}$ 一钛键，但是 这两个双键分处于环内与环外, 具有明显的差异, 因此 该化合物存在选择性切断两个相同类型化学键的潜在 可能.

\section{3 五元钛杂环}

在前过渡金属杂环化合物中，五元金属杂环化合物 相对较稳定, 例如已成功合成五元环炔(Scheme 17 中化 合物 32). 该类化合物主要是通过低价态金属与不饱和 化合物(如: 烯烃、炔烃、酮、醛、亚胺、腈等等)的偶 联反应得到 ${ }^{[50]}$. 由于这类金属杂环化合物具有两个活性 键, 因此它们能发生一系列的化学转化. 前过渡金属 (如: 铅、钛、铪)的金属环化反应具有相似的结构, 但也 存在着不同的反应模式与应用领域. 常见的五元钛杂环 的类型如 Scheme 17 所示, 由于钛杂环戊烷 29 易分解, 其相关研究较少 ${ }^{[17,51]}$.<smiles>CC1C(C)C(C)C(C)C1C</smiles>

29<smiles>CC1=C(C)C(C)C(C)C1C</smiles>

30<smiles>CC1=C(C)C(C)=C(C)C1=[Pt+2]</smiles>

31<smiles>CC1C=CC(C)C1C#N</smiles>

32
Scheme 17

\section{1 钛杂环戊烯}

在格氏试剂的还原体系下, $\mathrm{Cp}_{2} \mathrm{TiCl}_{2}$ 经格氏试剂还 原得到的钛-乙烯络合物 2a 可与炔烃发生插入反应, 生 成相应的钛杂环戊烯 $\mathbf{3 0}^{[20]}$, 反应中无副产物炔烃二聚 体生成, 可用于烯烃衍生物的合成(Scheme 18). 在类似 的条件下, 若采用 $\mathrm{Cp}_{2} \mathrm{ZrEt}_{2}$ 与一分子炔烃偶联, 会产生 $3 \% \sim 5 \%$ 的锆杂环戊二烯类化合物 ${ }^{[52]}$. 


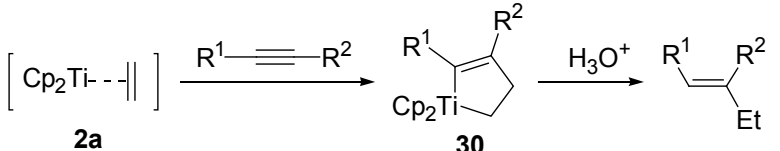

2a

30

Scheme 18

钛杂环戊烯 30 与一氧化碳的插入反应可高活性选 择地生成 $\alpha, \beta$-环戊酮类衍生物 $\mathbf{3 3}^{[53,54]}$ (Eq. 3).

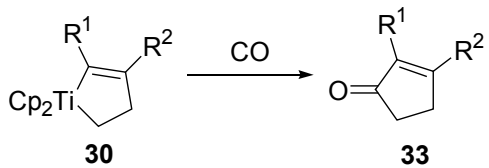

$\mathrm{R}^{1}=\mathrm{Ph}, n-\mathrm{Bu}, n-\mathrm{Pr} ;$

$\mathrm{R}^{2}=\mathrm{Ph}, n-\mathrm{Bu}, n-\mathrm{Pr}$, Me Yield $=63 \% \sim 90 \%$

上述反应中可能的机理是: 二茂钛活性物种 “ $\mathrm{Cp}_{2} \mathrm{Ti}$ ”上乙烯与炔烃经分子间还原偶联形成 30, CO 与 30 中的缺电子钛中心发生配位, 羰基插入 $\mathrm{C} \mathrm{C}_{\mathrm{sp}^{3}}$ 一钛键 中产生酰基化物 34, 34 经还原消除生成环戊烯酤 33, 同 时释放 “ $\mathrm{Cp}_{2} \mathrm{Ti}$ ” 活性物种进入下一个反应循环(Scheme $19)^{[55]}$

席振峰课题组 ${ }^{[56]}$ 发现钛杂环戊烯 30 与醛的反应体 现出与对应的锆杂环完全不同的性质. 锆杂环戊烯在 Lewis 酸的作用下与醛发生插入反应形成锆杂氧杂七元 环, 水解产物为高烯丙型氧代化合物 ${ }^{[57]}$, 而钛杂环戊

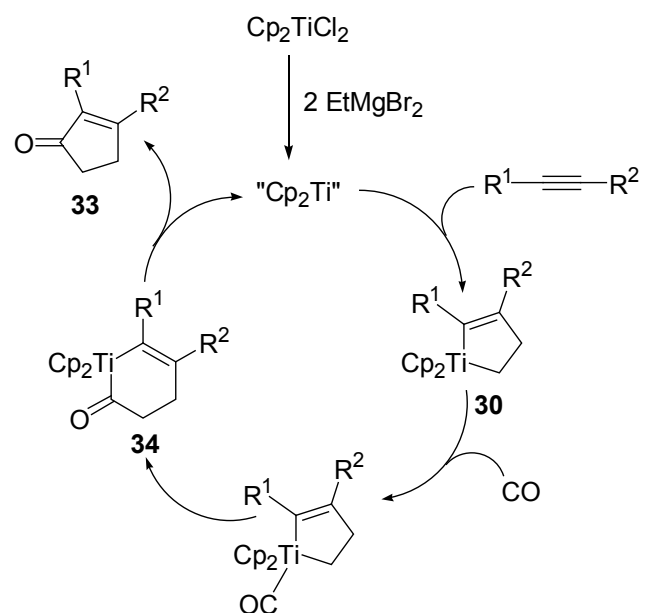

Scheme 19

烯在反应中并未形成钛杂氧杂七元环 35 , 而是通过配体 交换形成钛杂氧杂五元环 36, 该化合物经水解可生成烯 丙醇衍生物 37 (Scheme 20); 同样，钛杂环戊烯 30 与腈 的反应，也能经配体交换形成钛杂氮杂五元环 $\mathbf{3 8}$, 经水 解生成烯酩衍生物 39.

钛杂环戊烯 30 与醛的反应中，若体系存在 Lewis 酸，则反应选择性生成钛杂环戊烯 30 的水解产物 40. 当 在原位制备的钛杂氧杂五元环 36 体系中加入 Lewis 酸 时，经分子内取代反应令人意外地生成的 2,3-二苯基狮 衍生物 41 (Scheme 21).

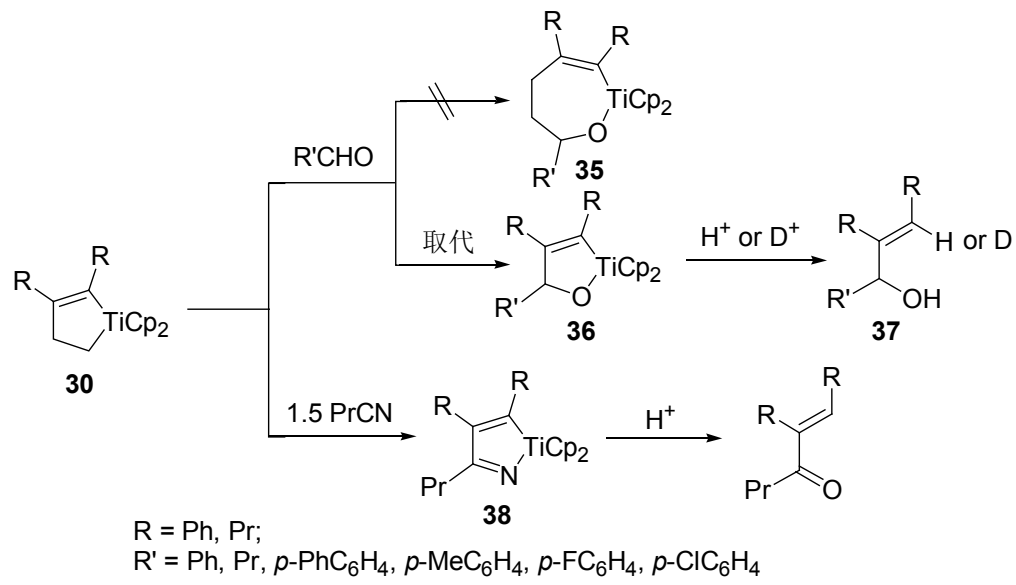

Scheme 20

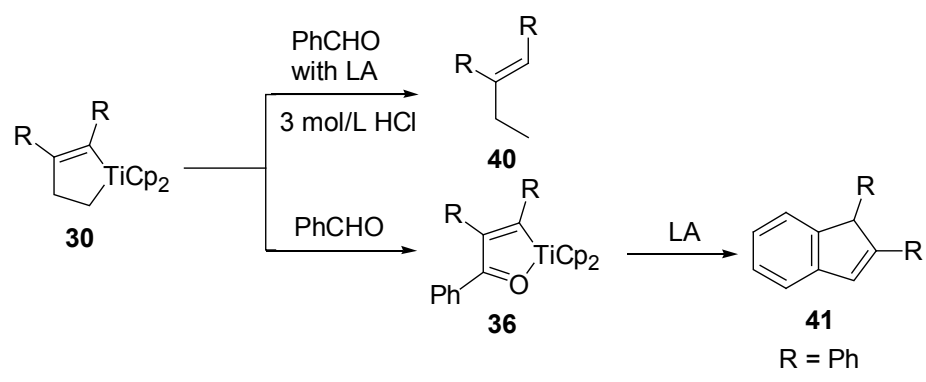

Scheme 21 
尽管在这些反应中 Lewis 酸的作用尚不明确, 但是 目前的研究结果表明 Lewis 酸与 “ $\mathrm{Cp}_{2} \mathrm{Ti}$ ” 之间的协同作 用是导致其异于锆杂环戊烯的反应的重要原因.

\section{2 钛杂环戊二烯}

在以 $n-\mathrm{BuLi}$ 为还原剂的还原体系下, 钛一烯烃络合 物 $\mathbf{2 b}$ 作为 “ $\mathrm{Cp}_{2} \mathrm{Ti}$ ” 等当体促进 2 分子炔烃发生还原偶 联反应生成钛杂环戊二烯 31 (Scheme 22).

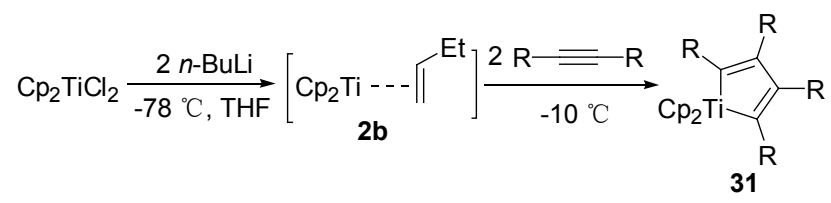

\section{Scheme 22}

该反应的反应机理可能有以下两条途径 ${ }^{[58]}$ (Scheme 23): 钛-丁烯络合物 $2 \mathbf{b}$ 作为 16 电子不稳定体系, 首先 与一分子炔烃发生插入反应得到 18 电子稳定结构中间 体 42, 可与炔烃发生配体交换得到中间体 43, 经插入反 应得到钛杂环戊二烯 31 (Path a); 或者钛-丁烯络合物 2b 首先与一分子炔烃发生配体交换得到 16 电子不稳定 中间体 44, 再吸收一分子炔烃形成中间体 43, 最后得到 31 (Path b). 目前钛杂环戊二烯通过哪种机理形成尚不 明确.

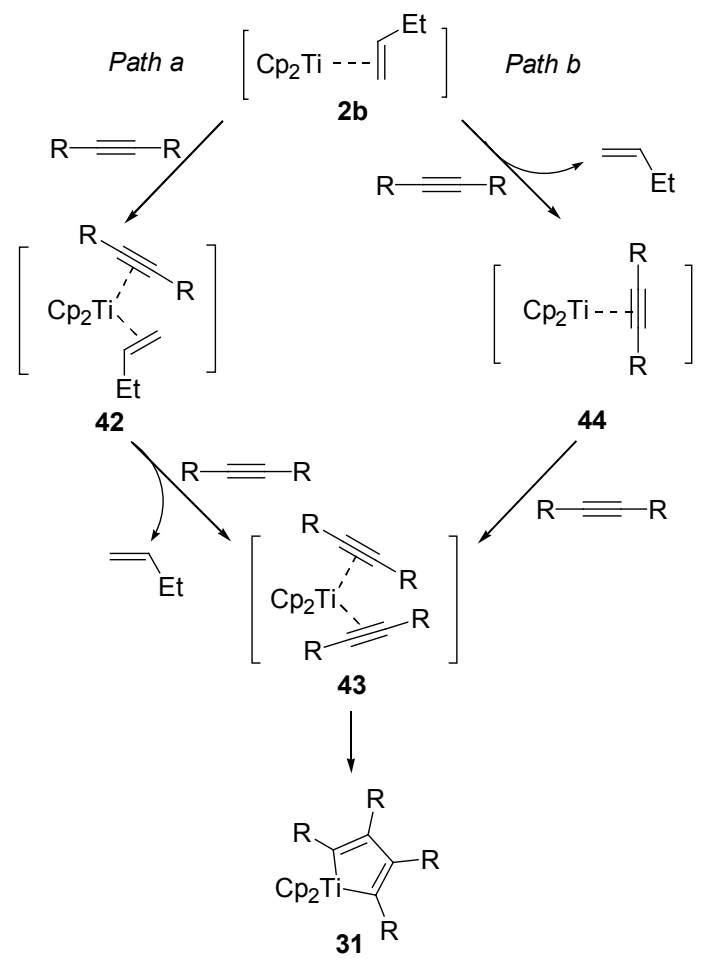

Scheme 23

化合物 31 中存在两个共轭的 $\mathrm{C}_{\mathrm{sp}^{2}}$ 一钛键, 具有令人 瞩目的反应活性，可用于多种类型化合物的合成，例如 强酸淬灭 31 可生成 1,3-丁二烯类衍生物(Eq. 4) ${ }^{[40]}$.

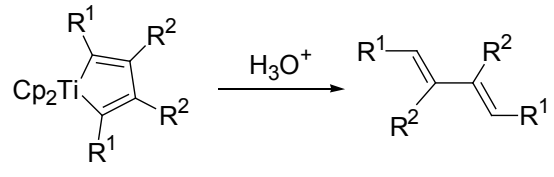

31

Takahashi 等 ${ }^{[59]}$ 在研究中发现，由两分子相同炔烃 形成的对称钛杂环成二烯在羧酸作用下，化合物 31 的 两个 $\mathrm{C}_{\mathrm{sp}^{2}}$ 一钛键可高选择地只断裂其中之一，另一个 $\mathrm{C}_{\mathrm{sp}^{2}}$ 一钛键可与新亲电试剂反应从而引入新基团, 为多 取代烯烃的合成提供新方法(Scheme 24).<smiles>[R]C=C([R])C([R])=C([R])[R]</smiles>

Scheme 24

在亲电试剂碘的作用下, 化合物 31 中的两个共轭 $\mathrm{C}_{\mathrm{sp}^{2}}$ 一钛键断裂形成相应的双碘代烯烃类衍生物 45. 在 催化剂的存在下, 该双碘代产物能进一步与芳香胺反 应，可用于构建多取代吡咯环骨架 ${ }^{[60]}$ (Scheme 25).

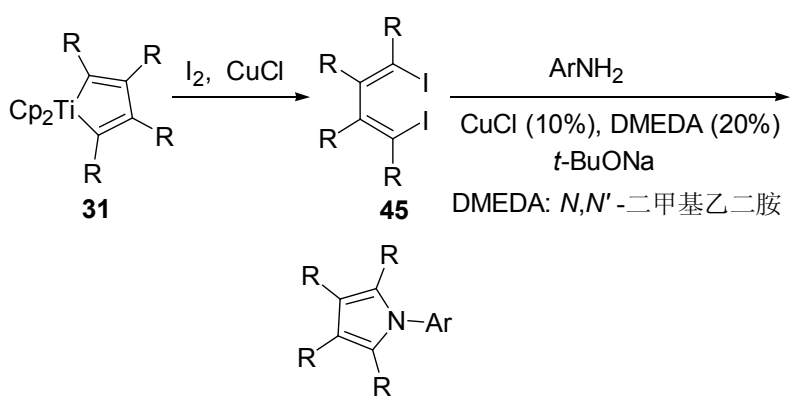

Scheme 25

共轭三烯类化合物具有较好的反应活性，并且其作 为一类多取代烯烃是构成天然化合物的重要骨架，许多 含有烯基的天然产物和化合物分子在生物药理方面展 现出特殊的作用. 在催化剂作用下, 31 可与第三分子炔 烃发生插入反应形成钛杂环庚三烯 46, 经水解形成 1,3,5-三烯类烯烃衍生物 ${ }^{[61]}$ (Scheme 26).

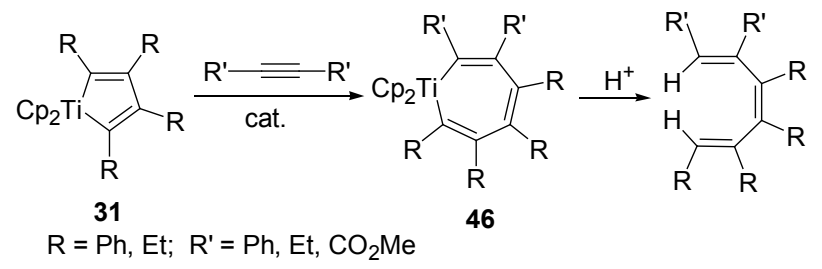

Scheme 26 
“C $\mathrm{p}_{2} \mathrm{Ti}$ ”活性物种除能与炔烃偶联外, 还能与联烯 化合物发生偶联反应. 1994 年 Doxsee 等 ${ }^{[62]}$ 首次报道了 钛试剂与联烯的反应，采用 $\mathrm{Cp}_{2} \mathrm{Ti}\left(\mathrm{PMe}_{3}\right)_{2}$ 与 3-甲基-1,2丁二烯反应，生成 $\alpha, \alpha^{\prime}$-双亚甲基钛杂环戍烷 47 (Eq. 5).

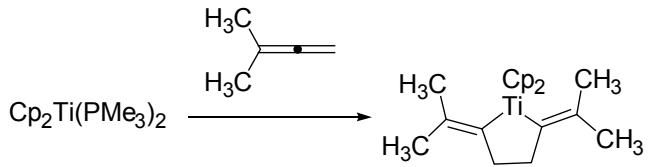

47
与钛杂环戊二烯 31 相比, 47 分子中同样存在两个 $\mathrm{C}_{\mathrm{sp}^{2}}$ 一钛键, 但是分子中两个双键是非共轭的, 存在异 于钛杂环戊二烯的潜在反应活性. 然而, 由于反应的选 择性受到联烯中取代基(取代基为烷基)个数及种类的影 响较大, 当 1,1-二取代联烯中取代基相同时选择性较好, 但其他类型底物反应的立体和区域选择性难题未被解 决, 至今研究较为有限.

上述反应中, 钛杂环化合物中成环的碳一钛键选择 性发生断裂，与多种亲电试剂反应形成各种类型的化合 物. 在这些反应中, 金属配合物中茂环作为金属中心的 配体而存在. 近年来研究结果表明, 与钛相连的茂环除 了作为配体之外，也可以参与产物骨架的构建.

在 Scheme 9 中我们曾经提到钛杂环戊二烯 11 可在 $-20{ }^{\circ} \mathrm{C}$ 时形成, 当温度上升至 $0{ }^{\circ} \mathrm{C}$ 时钛上的一个 $\mathrm{Cp}$ 环 发生断裂, 参与钛杂环的偶联反应形成二氢狮 $\pi$-配合物 48, 经还原消除形成䒢衍生物 $4^{[29]}$ (Scheme 27).

当化合物 6 与双硼酸频呐醇酯基乙炔反应时，生成 的产物是两分子炔烃经还原偶联后与钛的其中一个配 体环戍二烯形成六元环并五元环的物种 23 . 当钛中心的 配体改用体积更大的 $\mathrm{Cp}^{*}\left(\mathrm{Cp}^{*}=\eta^{5}\right.$-五甲基环戊二烯基) 时配体不再参与反应，依据不同反应时长可选择性形成 双硼酸酯基钛杂环丙烯或四硼酸酯基钛杂环戊二烯 ${ }^{[63]}$. 由此可见，金属钛上的配体 $\mathrm{Cp}$ 环并非是隋性的.

Takahashi 等 ${ }^{[64]}$ 在对钛杂环戊二烯 31 的研究中发现, 与钛相连的两个 $\mathrm{Cp}$ 环中的其中一个 $\mathrm{Cp}$ 环除会发生裂解 外，五元钛杂环上的烷基会发生迁移，形成中间体 $\mathbf{5 0}$,

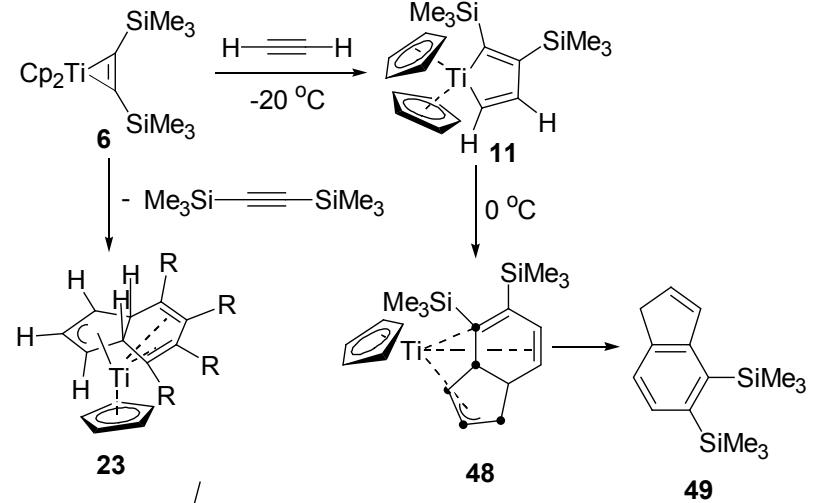

Scheme 27

与中间体 $\mathbf{5 1}$ 达到动态平衡从而形成 $\mathrm{R}$ 基迁移的狮衍生 物 52 (Scheme 28); 而在偶氮苯的作用下，中间体 $\mathbf{5 0}$ 末 经形成与之平衡的中间体 51, 而直接形成狮衍生物 53 .

在铜盐促进下, 钛杂环戊二烯 31 与腈反应, 31 中与 金属钛相连的 2 个 $\mathrm{Cp}$ 环的其中一个被 “撕裂”为 2 部 分，其中 “3 碳” 部分与 2 分子腈生成吡啶类衍生物 54, 而“2 碳”部分则与钛相连的环戊烯结合生成苯衍生物 $\mathbf{5 5}^{[65]}$ (Eq. 6).

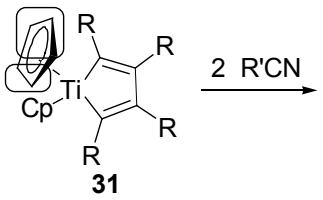

此外，钛杂环戊二烯 31 中与钛相连的 $\mathrm{Cp}$ 环除能发 生裂解外, $\mathrm{Cp}$ 环可整体作为亲电试剂进行反应 ${ }^{[59]}$. 由钛 杂环戊烯 31 选择性单质子化形成的烯共轭烯基钛中间 体中，受到钛原子上配体之间位阻效应的驱动，其中一 个 $\mathrm{Cp}$ 环作为亲电试剂与烯基形成新的碳碳键生成 56 . 而在偶氮苯的作用下, 56 的产率可由原来的 $38 \%$ 提高到 $92 \%(\mathrm{R}=\mathrm{Ph})($ Scheme 29).

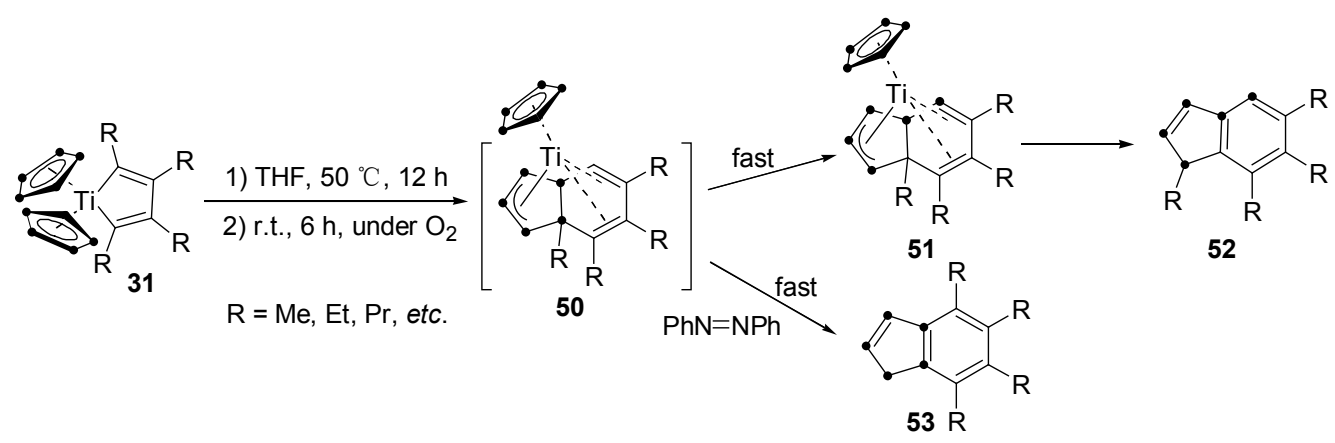

Scheme 28 


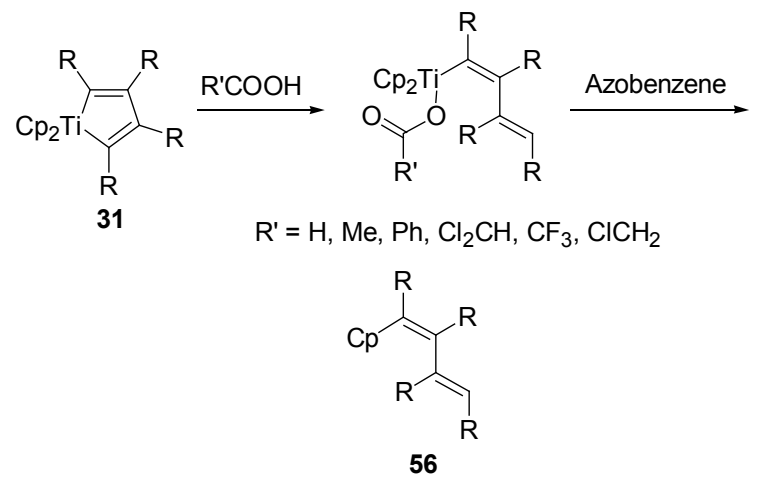

Scheme 29

虽然在钛杂环戊二烯和锆杂环戊二烯中均具有两 个共轭 $\mathrm{C}_{\mathrm{sp}^{2}}$ 一金属键, 但两者表现出较大的反应活性差 异, 对钛杂环戍二烯具备的潜在反应活性的研究具有重 要的理论意义和应用价值.

\section{3 钛杂环戊-3-炔}

小环炔烃作为一种张力环是非常不稳定而较难分 离 ${ }^{[6]}$, 其中五元环炔具有较高的反应活性, 寿命较短. 钛杂环戊-3-炔可通过图 36 中两种方式合成, 即 1,4-二 氯-2-丁炔或 1,4-二硅基-1,2,3-丁三烯分别与二价钛物种 反应生成无取代钛杂环戊炔 32a 及 $\alpha, \alpha^{\prime}$-二硅基铁杂环戊 炔 32b. 钛 $\alpha$-位引入硅基对负电荷的稳定化作用使 32b 的核磁产率略高, 但是这两种化合物分离产率分别是 $9 \%$ 与痕量. 产物 32a 与 $32 \mathrm{~b}$ 可通过原位光谱法确证 ${ }^{[67]}$.

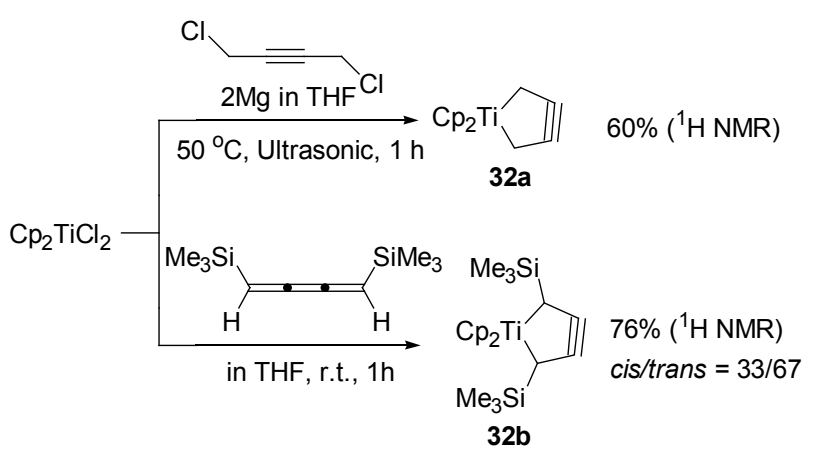

Scheme 30

\section{4 六元钛杂环化合物}

六元钛杂环化合物的合成主要有以下两种方法 ${ }^{[4,68]}$. 首先, 钛杂环己烷 57 可由 $\mathrm{Cp}_{2} \mathrm{TiCl}_{2}$ 经 1,6-二锂已烷还原 脱氯制得(Eq. 7).

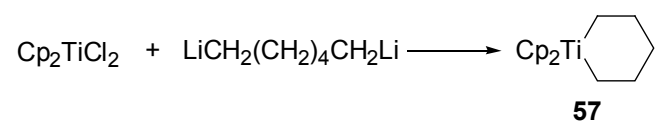

此外, $\mathrm{Cp}_{2} \mathrm{TiCl}_{2}$ 与 2,2'-二锂联苯反应可生产相应的
二苯骈钛杂环己二烯化合物 58 (Eq. 8). 目前对六元钛 杂环化合物的报道较少，其多数以反应中间体的形式存 在 $^{[56,69]}$.

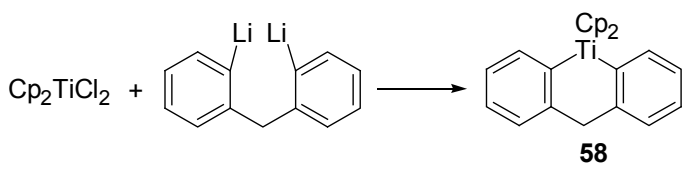

由于合成方法的欠缺，对六元钛杂环化合物的合成 及潜在活性研究是一大挑战.

\section{5 结论与展望}

综上所述, 钛杂环化合物从三元环到六元环, 种类 繁多, 其反应活性与同族元素铅杂环相比有明显差异, 分子中的两个碳金属键为选择性化学转化提供多种途 径, 是一类有用的有机合成中间体. 其中用于构建钛杂 环骨架的不饱和体系包括烯烃、炔烃、羰基化合物、亚 胺等, 然而尚有一些 $\pi$ 体系的研究较少, 例如联烯. 基于 此, 我们相信开展新型钛杂环的合成及应用研究充满着 机遇与挑战, 对发展钛化学及实现一些新的合成方法学 具有一定的理论意义及应用价值.

\section{References}

[1] (a) Pramod, R. C.; Janis, L. Adv. Synth. Catal. 2006, 348, 2307.

(b) Jesu's, A. V.; Carlos, S. Chem. Rev. 2003, 103, 3787.

(c) Shinichi, S.; Yoshinori, Y. Chem. Rev. 2000, 100, 2901.

(d) Iwao, O.; Maria, T.; Li, Z. Y.; Robert, J. D. Chem. Rev. 1996, 96, 635.

(e) Itaru, N.; Yoshinori, Y. Chem. Rev. 2004, 104, 2127.

(f) Zhang, C. H.; Zhang, H.; Wei, A. L.; He, X. M. Acta Chim Sinica 2013, 71, 1373 (in Chinese).

(张春红, 张弘, 魏爱琳, 何旭敏, 夏海平, 化学学报, 2013, 71, 1373.)

[2] (a) Gerald, D. Angew. Chem., Int. Ed. 1999, 38, 1699.

(b) Shinich, I. Organomet. News 2007, 1, 2.

(c) Kochi, J. K. ACS Symposium Series, Vol. 55, Oxford University Press, New York, 1977, p. 167.

[3] (a) Tarselli, M. A. Nat. Chem. 2013, 5, 546.

(b) Duthaler, R. O.; Hafner, A. Chem. Rev. 1992, 92, 807.

(c) Reetz, M. T. Acc. Chem. Res. 1993, 26, 462.

[4] (a) Dunitz, J. D.; Orgel, L. E. Nature 1953, 171, 121. (b) Dunitz, J. D.; Orgel, L. E. J. Chem. Phys. 1955, 23, 954.

[5] Piotr, S.; Anna, D. J.; Jozef, U.; Lucjan, B. J. Organometallics 2011, $30,1741$.

[6] Xi, Z. F.; Li, Z. P. Top. Organomet. Chem. 2004, 8, 27.

[7] Rosebthal, U.; Vladimir, V. B. In Titanium and Zirconium in Organic Synethesis, Ed.: Marek, I., Wiley-VCH, 2002, p. 355.

[8] Fischer, A. K.; Wilkinson, G. J. Inorg. Nucl. Chem. 1956, 2, 149.

[9] Chirik, P. J. Organometallics 2010, 29, 1500.

[10] Eisch, J. J.; Adeosun, A. A.; Birmingham, J. M. Eur. J. Inorg. Chem. 2007, 1, 39.

[11] Watt, G. W.; Drummond, F. O. J. Am. Chem. Soc. 1970, 92, 826.

[12] Freitag, M. A.; Gordon, M. S. J. Phys. Chem. A 2002, 106, 7921.

[13] (a) Liu, C. F.; Chen, J. H.; Chen, S. C.; Chen, K. Y.; Lee, G. H.; Peng, S. M. J. Org. Chem. 2005, 690, 291. 
(b) Timothy, J. C.; Christopher, A. R.; Ian, M. J. Am. Chem. Soc. 2006, 128,9582 .

[14] (a) Xu, S.; Tao, X-C. Acta Chim. Sinica 2004, 24, 250 (in Chinese) (许胜, 陶晓春, 化学学报, 2004, 24, 250.)

(b) Fumie, S.; Hiroaki, I.; Masao, S. Tetrahedron Lett.1981, 22, 85.

[15] (a) Bonnemann, O. Angew. Chem., Int. Ed. Engl. 1985, 24, 248.

(b) Vollhardt, K. P. C. Angew. Chem., Int. Ed. Engl. 1984, 23, 539.

(c) Takeda, T.; Tsubouchi, A. J. Synth. Org. Chem. 2008, 66, 1178.

[16] Li, Z. P.; Zhao, P. J.; Xi, Z. F. Chemistry 2002, (2), 78 (in Chinese). (李志平, 赵炳筠, 席振峰, 化学通报, 2002, (2), 78.)

[17] (a) Negishi, E.; Huo, S. Q. In Titanium and Zirconium in Organic Synethesis, Ed.: Marek, I., Wiely-VCH, 2002, p. 1.

(b) Takahashi, T.; Li, Y. Z. In Titanium and Zirconium in Organic Synethesis, Ed.: Marek, I., Wiely-VCH, 2002, p. 50.

[18] (a) Takahashi, T.; Murakami, M.; Kunishige, M.; Saburi, M.; Uchida, Y.; Kozawa, K.; Uchida, T.; Swanson, D. R.; Negishi, E. Chem. Lett. 1989, 5, 761.

(b) Takahashi, T.; Takashi, S.; Nitto, Y.; Masahiko, S.; Christophe, J. R.; Negishi, E. J. Am. Chem. Soc. 1991, 113, 6266.

(c) Takahashi, T.; Suzuki, N.; Kageyama, M.; Nitto, Y.; Saburi, M.; Negishi, E. Chem. Lett. 1991, 9, 1579.

(d) Takahashi, T.; Kageyama, M.; Denisov, V.; Hara, R.; Negishi, E. Tetrahedron Lett. 1993, 34, 687.

(e) Takahashi, T.; Xi, C. J.; Xi, Z. F.; Kageyama, M.; Fischer, R.; Nakajima, K.; Negishi, E. J. Org. Chem. 1998, 63, 6802.

[19] Negishi, E.; Takahashi, T. Synthesis 1988, 1.

[20] Negishi, E.; Fredrik, E. C.; Takahashi, T. Tetrahedron Lett. 1986, 27, 2829.

[21] (a) Nicolas, M.; Narcis, A.; Didier, B.; Francois, M.; Pascal, L. F. Organometallics 1998, 17, 2677.

(b) Shur, V. B.; Berkovich, E. G.; Volpin, M. E. J. Organomet. Chem. 1982, 228, 36.

(c) Shur, V. B.; Burlakov, V. V.; Volpin, M. E. J. Organomet. Chem. 1988, 347, 77 .

(d) Fumie, S.; Hirokazu, U. In Titanium and Zirconium in Organic Synethesis, Ed.: Marek, I., Wiely-VCH, 2002, p. 319.

[22] Fagan, P. J.; Nugent, W. A. J. Am. Chem. Soc. 1988, 110, 2310.

[23] (a) Uwe, R.; Vladimir, V. B.; Marc, A. B.; Torsten, B. Chem. Soc. Rev. 2007, 36, 719.

(b) Uwe, R.; Vladimir, V. B.; Perdita, A.; Wolfgang, B.; Anke, S. Organometallics 2005, 24, 456.

[24] Kaleta, K.; Ruhmann, M.; Theilmann, O.; Beweries, T.; Roy, S.; Arndt, P.; Villinger, A.; Eluvathingal, D. J.; Schulz, A.; Rosenthal, U. J. Am. Chem. Soc. 2011, 133, 5463.

[25] (a) Leigh, G. Chem. Eur. J. 1997, 3, 332.

(b) Uwe, R.; Vladimir, V.; Burlakov, P. A.; Wolfgang, B.; Anke, S. Organometallics 2003, 22, 884.

[26] Martin, L.; Anke, S.; Wolfgang, B.; Jiao, H. J.; Christine, F.; Sven, H.; Perdita, A.; Uwe, R. J. Am. Chem. Soc. 2010, 132, 43690.

[27] (a) Uwe, R.; Vladimir, V. B. In Titanium and Zirconium in Organic Synethesis, Ed.: Marek, I., Wiely-VCH, 2002, p. 355.

(b) Bradford, C.; Wagenen, V.; Livinghouse, T. Tetrahedron Lett. 1989, 30, 3495 .

[28] Lefeber, C.; Ohff, A.; Tillack, A.; Baumann, W.; Kempe, R.; Burlakov, V. V.; Rosenthal, U.; Görls, H. J. Organomet. Chem. 1995, 501, 179.

[29] Thomas, D.; Peulecke, N.; Burlakov, V. V.; Heller, B.; Baumann, W.; Spannenberg, A.; Kempe, R.; Rosenthal, U.; Beckhaus, R. Z. Anorg. Allg. Chem. 1998, 624, 919.

[30] Roy, S.; Jemmis, E. D.; Schulz, A; Beweries, T.; Rosenthal, U. Angew. Chem., Int. Ed. 2012, 51, 5347.

[31] (a) Rosenthal, U.; Burlakov, V. V.; Bach, M. A.; Beweries, T. Chem.
Soc. Rev. 2007, 36, 719 .

(b) Lamac, M.; Spannenberg, A.; Jiao, H.; Hansen, S.; Baumann, W.; Arndt, P.; Rosenthal, U. Angew. Chem., Int. Ed. 2010, 49, 2973.

(c) Becker, L.; Burlakov, V. V.; Arndt, P.; Spannenberg, A.; Baumann, W.; Jiao, H. J.; Rosenthal, U. Chem.-Eur. J. 2013, 19, 4230 .

(d) Oishi, S.; Tsubouchi, A.; Takeda, T. J. Organomet. Chem. 2012, 696, 581 .

[32] (a) Takeda, T. Bull. Chem. Soc. Jpn. 2005, 78, 195.

(b) Bernhard, B. Angew. Chem., Int. Ed. Engl. 1998, 37, 453.

[33] Tebbe, F. N.; Parshall, G. W.; Reddy, G. S. J. Am. Chem. Soc. 1978, $100,3611$.

[34] (a) Petasis, N. A.; Bzowej, E. I. J. Am. Chem. Soc. 1990, 112, 6392. (b) Petasis, N. A.; Lu, S.P.; Bzowej, E. I.; Fu, D.K.; Staszewski, J. P.; Akritopoulou-Zanze, I.; Patane, M. A.; Hu, Y. H. Pure Appl. Chem. 1996, 68, 667.

(c) Vaxelaire, C. Synlett 2009, 3221.

(d) Wu, K.; Mercado, E. V.; Pettus, T. R. R. J. Am. Chem. Soc. 2011, 133, 6114 .

[35] Theilmann, O.; Ruhmann, M.; Villinger, A.; Schulz, A.; Wolfram, W. S. Angew. Chem., Int. Ed. 2010, 49, 9282.

[36] Beckhaus, R.; Santamarı'a, C. J. Organomet. Chem. 2001, 617, 81.

[37] Takeda, T. Chem. Rec. 2007, 7, 24.

[38] (a) Tebbe, F. N.; Parshall, G. W.; Ovenall, D. W. J. Am. Chem. Soc 1979, 101, 5074.

(b) Howard, T. R.; Lee, J. B.; Grubbs, R. H. J. Am. Chem. Soc. 1980, 102,6876 .

(c) Lee, J. B.; Kevin, C. O.; Grubbs, R. H. J. Am. Chem. Soc. 1982, 104, 7491 .

[39] John, R. S.; Bernard, D. S.; Robert, H. G. J. Org. Chem. 1990, 55, 843.

[40] Takahashi, T. In Titanium and Zirconium in Organic Synethesis, Ed.: Marek, I., Wiely-VCH, 2002, p. 475.

[41] (a) Gilliom, L. R.; Grubbs, R. H. Organometallics 1986, 5, 721. (b) Stille, J. R.; Grubbs, R. H. J. Am. Chem. Soc. 1986, 108, 855.

[42] (a) Hawkins, J. M.; Grubbs, R. H. J. Am. Chem. Soc. 1988, 110, 2821.

(b) Beckhaus, R.; Wagner, T. J. Organomet. Chem. 1993, 460, 181.

(c) Stephen, L. B.; Robert, H. G. J. Am. Chem. Soc.1983, 105, 5490.

[43] (a) Kenneth, M. D.; Jerrick, J. J. J.; Kenneth, Z.; Gregory, N. J. Am. Chem. Soc. 1994, 116, 2147.

(b) Petasis, N. A.; Fu, D. K. Organometallics 1993, 12, 3776.

[44] Meinhart, J. D.; Grubbs, R. H. Bull. Chem. Soc. Jpn. 1988, 61, 171.

[45] Kenneth, M. D.; John, K. M. M. Tetrahedron Lett. 1991, 32, 1687.

[46] Shono, T.; Nagasawa, T.; Tsubouchi, A.; Takeda, T. Tetrahedron Lett. 2007, 48, 3521 .

[47] Shono, T.; Nagasawa, T.; Tsubouchi, A.; Noguchi, K.; Takeda, T. Chem. Commun. 2008, 3537.

[48] Shono, T.; Kurashige, R.; Mukaiyama, R.; Tsubouchi, A.; Takeda, T. Chem. Eur. J. 2007, 13, 4074

[49] Yatsumonji, Y.; Atake, Y.; Tsubouchi, A.; Takeda, T. Chem. Commun. 2009, 3375.

[50] (a) Fumie, S.; Hirokazu, U.; Sentaro, O. Chem. Rev. 2000, 100, 2835.

(b) Negishi, E.; Takahashi, T. Acc. Chem. Res. 1994, 27, 124.

[51] McDermott, J. X.; Wilson, M. E.; Whitesides, G. M. J. Am. Chem. Soc. 1976, 98, 6529 .

[52] (a) Takahashi, T.; Kageyama, M.; Denisov, V.; Hara, R.; Negishi, E. Tetrahedron Lett. 1993, 34, 687.

(b) Takahashi, T.; Xi, Z. F.; Nishihara, Y.; Huo, S.; Kasai, K.; Aoyagi, K.; Denisov, V.; Negishi, E. Tetrahedron 1997, 53, 912.

[53] Kimihiko, S.; Yasushi, N.; Huo, S. Q.; Xi, Z. F.; Takahashi, T. J. 
Organomet. Chem. 2001, 633, 18.

[54] Zhao, Z. B.; Ding, Y.; Zhao, G. J. Org. Chem. 1998, 63, 9285.

[55] Frederick, A. H.; Natasha, M. K.; Buchwald, L. S. J. Am. Chem. Soc. 1999, 121, 5881.

[56] (a) Zhao, C.; Yu, T.; Xi, Z. Chem. Commun. 2002, 2, 142.

(b) Zhao, C.; Yan, J.; Xi, Z. J. Org. Chem. 2003, 68, 4355.

(c) Zhao, C.; Lu, J.; Yan, J.; Xi, Z. Tetrahedron Lett. 2003, 44, 6895.

(d) Zhao, C.; Lu, J.; Li, Z.; Xi, Z. Tetrahedron 2004, 60, 1417.

[57] (a) Coperet, C.; Negishi, E.; Xi, Z.; Takahashi, T. Tetrahedron Lett. 1994, 35, 695.

(b) Li, P.; Xi, Z.; Takahashi, T. Chin. J. Chem. 2001, 19, 45.

[58] Xi, Z. F.; Hara, R.; Takahashi, T. J. Org. Chem. 1995, 60, 4444.

[59] Song, Z. Y.; Hsieh, Y. F.; Kanno, K.; Nakajima, K.; Takahashi, T. Organometallics 2011, 30, 844.

[60] Liao, Q.; Zhang, L.; Wang, F.; Li, S.; Xi, C. J. Eur. J. Org. Chem. 2010, 28, 5426 .

[61] Famili, A.; Farona, M. F.; Thanedar, S. J. Chem. Soc., Chem. Commun. 1983, 3, 435.

[62] Doxsee, K. M.; Juliette, J. J. J.; Zientara, K.; Nieckarz, G. J. Am. Chem. Soc. 1994, 116, 2147.

[63] Altenburger, K.; Arndt, P.; Spannenberg, A.; Baumann, W.; Rosenthal, U. Eur. J. Inorg. Chem. 2013, 3200.

[64] (a) Takahashi, T.; Song, Z. Y.; Sato, K.; Kuzuba, Y.; Nakajima, K.;
Ken-ichiro, K. J. Am. Chem. Soc. 2007, 129, 11678.

(b) Takahashi, T.; Kuzuba, Y. C.; Kong, F. Z.; Nakajima, K.; Xi, Z. F. J. Am. Chem. Soc. 2005, 127, 17188.

(c) Takahashi, T.; Song, Z. Y.; Hsieh, Y. F.; Nakajima, K.; Ken-ichiro, K. J. Am. Chem. Soc. 2008, 130, 15236.

[65] (a) Xi, Z. F.; Kimihiko, S.; Gao, Y.; Lu, J. M.; Tamotsu, T. J. Am. Chem. Soc. 2003, 125, 9568.

(b) Kempe, R. Angew. Chem., Int. Ed. 2004, 43, 1463.

(c) Takahashi, T.; Kanno, K. Organomet. Chem. 2004, 8, 217.

(d) Xi, Z. F.; Kong, F. Z.; Zheng, W. X.; Zhang, W. X. Chin. J. Org. Chem. 2005, 25, 229 (in Chinese).

(席振峰, 孔凡志, 郑卫新, 张文雄, 有机化学, 2005, 25, 229.)

[66] (a) Gleiter, R.; Merger, In Mordern Acetylene Chemistry, Eds.: Stang, P. J.; Diederich, F., Wiley-VCH, 1995, Chapter 8.

(b) Meier, H. Adv. Strain Org. Chem. 1991, 1, 215.

[67] (a) Suzuki, N.; Watanabe, T.; Yoshida, H.; Iwasaki, M.; Saburi, M.; Tezuka, M.; Hirose, T.; Hashizume, D.; Chihara, T. J. Organomet. Chem. 2006, 691, 1175.

(b) Bolster, J. M.; Kellogg, R. M. J. Am. Chem. Soc. 1981, 103, 2868.

[68] (a) Carl, A. M.; Todd, N.; Graham, J.; Cordes, A. W.; Allison, N. T. Organometallics 1988, 7, 2573.

(b) Robert, H. G.; Akira, M. J. Am. Chem. Soc. 1978, 100, 7418 .

[69] Crowe, W. E.; Vu, A. T. J. Am. Chem. Soc. 1996, 118, 1557.

(Cheng, F.) 